\title{
Estudio de la inteligencia emocional en escolares de educación primaria: Alcance en el área de educación física
}

\section{Study of emotional intelligence in primary school: Scope in the area of physical education}

\author{
Alejandro Gabriel Sánchez Ortega* y Ramón Chacón-Cuberos \\ Facultad de Ciencias de la Educación, Universidad de Granada (España).
}

\begin{abstract}
Resumen: La Inteligencia Emocional (IE) se puede considerar una habilidad para conseguir un aprendizaje más significativo, si se lleva a cabo durante los procesos de enseńanza-aprendizaje. Esto es así, ya que, por cada situación que aparezca en el aula durante los procesos de enseńanza, conlleva una o varias emociones desatadas por esas situaciones, y es aquí donde el docente puede reforzar de forma positiva y significativa las dimensiones de esa emoción u emociones surgidas. Tener equilibrio emocional significa tener un buen bienestar físico y mental, puesto que cuanto más saludables sean nuestras emociones, mejor nos vamos a sentir en diferentes situaciones diarias. Por otro lado, realizar una Actividad Física (AF) o cualquier deporte, es beneficioso para el bienestar físico y mental, ya que, realizar actividad física conlleva una serie de emociones positivas en los individuos que la practiquen. Al igual, que emociones positivas, la $\mathrm{AF}$ produce emociones negativas, por lo que hemos optado por reforzar y contrarrestar estas emociones durante la práctica de Educación Física (EF) en Educación Primaria, puesto que cuánto antes se redirijan estas emociones, mejor equilibrio emocional y mejor aprendizaje se va a conseguir, y como mejor que realizarlo a partir del área de EF, y promovido por un docente.

Palabras clave: Inteligencia Emocional; Educación Primaria; Escolares; Educación Física
\end{abstract}

Abstract: Emotional Intelligence (EI) can be considered an ability to achieve more meaningful learning, if carried out during the teaching-learning processes. This is so, since, for each situation that appears in the classroom during the teaching processes, it involves one or several emotions unleashed by those situations, and it is here that the teacher can positively and significantly reinforce the dimensions of that emotion or emotions arisen. Having emotional balance means having a good physical and mental well-being, since the healthier our emotions are, the better we will feel in different daily situations. On the other hand, performing a Physical Activity (AF) or any sport is beneficial for physical and mental well-being, since physical activity involves a series of positive emotions in the individuals who practice it. Like positive emotions, FA produces negative emotions, so we have chosen to reinforce and counteract these emotions during the practice of Physical Education (PE) in Primary Education, since the sooner these emotions are redirected, the better emotional balance and better learning is going to be achieved, and as better than to be done from the area of EF, and promoted by a teacher.

Keywords: Emotional Intelligence; Primary Education; School-children; Physical Education.

\section{Introducción}

El concepto de "Inteligencia emocional" (IE) se define como la habilidad para percibir, asimilar, comprender y regular las emociones propias y ajenas, promoviendo un crecimiento emocional e intelectual. Salovey y Mayer (1990), consideraban dentro del concepto "Inteligencia Emocional" cuatro dimensiones básicas: percepción emocional (identificar y reconocer), facilitación emocional (facilitar y activación de pensamientos), comprensión emocional (interpretar diferentes emociones y estimación de futuras consecuencias) y regulación emocional (capacidad de estar abierto a los sentimientos y reflexionar sobre ellos). Por otro lado, también podemos dar a conocer conceptos muy importantes dentro de la IE como a raíz de trabajos posteriores como el desarrollado por García, Graupera, Ruiz y Palomo, 2013 (Zurita-Ortega et al., 2018). Entre estos se encuentra la "Gestión auto-emocional", "Ges-

Dirección para correspondencia [Correspondence address]: Alejandro Gabriel Sánchez Ortega. Universidad de Granada (España).

Email: alexalfacar1@gmail.com tión hetero-emocional" y "Utilización emocional". La "Gestión auto-emocional", es saber gestionar, controlar y manejar nuestras propias emociones. "Gestión hetero-emocional" se relaciona bastante con el término anterior, pero en este caso es saber gestionar, controlar y manejar distintas emociones surgidas de situaciones externas al sujeto. Por último, "Utilización emocional" se vincularía al cómo usar las emociones que surjan en cualquier proceso o actividad. Estos conceptos van a ser transcendentales para el desarrollo de este trabajo, relacionando así tanto la actividad física como la IE.

Para educar las emociones se necesita preparación, experiencia y tiempo. Debemos profundizar en todo tipo de problemas que surjan en el aula, reconociendo así diferentes emociones producidas para conseguir un bien común y así una solución para este problema o comportamiento (ArangoCaramelo, 2017). Este tipo de actuaciones en el aula hacen reflexionar a los alumnos/as sobre lo correcto y lo incorrecto, el beneficio y el prejuicio, lo cómodo y lo incómodo... La educación emocional debe ser un proceso continuo y permanen- 
te para poder desarrollarlo adecuadamente y correctamente, con un fin en común de prevenir (Bisquerra, 2005). Por este motivo debemos trabajarlo con alumnos/as con problemas de conducta, que es precisamente un factor educativo que debemos evitar (Arango-Caramelo, 2017).

La Actividad Física (AF) y la IE pueden generar altos niveles de bienestar físico, mental, emocional y social, por lo que es importante trabajar estos factores desde edades tempranas, por medio de programas específicos en el área de EF (De Benito, 2013). No obstante, también puede suceder al contrario (Peña, 2019). Por lo que, la etapa de Educación Primaria es primordial, por la cantidad de estímulos que abundan en esta etapa tanto social como familiar son la definición en gran medida de capacidades vitalicias de aprendizaje (Conde y Almagro, 2015).

La Educación Física (EF) genera diversas situaciones donde se ponen en juego habilidades sociales, y de ahí surgen diferentes emociones entre los alumnos, proporcionando a estos la regulación de diferentes emociones puestas en práctica, y al docente de EF la posibilidad de mostrar estrategias de regulación de estas emociones, como la resolución de posibles conflictos, el autocontrol, etc. (Kim, Khon, Aidosova, 2016; McPhie y Rawana, 2015; Tillmann, Krishnadas, Cavanagh y Petrides, 2013).

El juego, tiene papel fundamental sobre la reacción emocional particular, contando con altos niveles de alegría y buen humor (Duran, Lavega, Salas, Tamarit e Invernó, 2015). La IE fomenta la creatividad, o viceversa, por lo que para un alumno/a, el diseñar una actividad con fin lúdico donde esté presente la actividad física supone una evolución psicológica para la creatividad del alumno/a creador y una reacción emocional positiva, tanto individual como general por parte del alumnado durante la práctica de esta actividad física (Lleixá, 2017). La práctica de actividad física voluntaria relacionada con la expresión corporal, despliegan capacidades de atención, claridad y reparación emocional del alumnado, favoreciendo la capacidad de expresión, el conocimiento de uno mismo y la comprensión de los demás, es decir, facilitando la habilidad interpersonal e intrapersonal, contribuyendo a la reparación de los estados emocionales negativos (Guszkowska, Méndez, Martínez de Ojeda y Valverde, 2017; Salguero, 2010). En base a todo lo expuesto, el presente Trabajo de Fin de Grado persigue como principales objetivos:

- Describir niveles de la Actividad Física en diferentes dimensiones de la Inteligencia Emocional en alumnos de tercer ciclo de Educación Primaria, en concreto en el área de EF.

- Analizar las relaciones entre la práctica de Actividad Física, la Inteligencia Emocional y diferentes factores sociodemográficos.

\section{Material y método}

\subsection{Diseño y Participantes}

Se presenta un estudio no experimental, de tipo descriptivo y corte transversal. El número de participantes es de 86 estudiantes de Educación Primaria. La muestra estuvo comprendida entre edades 10 a los 11 con una edad media de $10,87 \pm 0,33$, donde el $43 \%(n=37)$ corresponde a los hombres y un $57 \%(n=49)$ a mujeres. El criterio de selección de estos participantes fue que estuvieran cursando sexto curso de Educación Primaria.

\subsection{Instrumentos-Variables}

Se empleó el Inventario de Inteligencia Emocional en el Deporte de García, Graupera, Ruiz y Palomo (2013). Este instrumento fue desarrollado para dos grupos de chicos/as con una edad entre 10-11 años y su validación ofreció una solución unifactorial, con un cuestionario que se divide en 30 ítems sobre la IE, en las que se obtienen 4 dimensiones: Inteligencia Emocional Global (Esta dimensión es la nota ponderada en la escala de Likert de cada muestra, de las siguientes dimensiones), Percepción Emocional (e.g. 2.Cuándo tengo un problema, recuerdo antiguos problemas parecidos que tuve y cómo los superé), Gestión Auto-Emocional (e.g. 25.Soy consciente de los gestos que envío a los/as otros/as compañeros/as), Gestión Hetero-Emocional (e.g. 6.Algunos de los acontecimientos más importantes de mi vida me han provocado pensar en qué es lo realmente importante y lo qué no) y Utilización Emocional (e.g. 7.Cuando mi estado de ánimo cambia, veo nuevas posibilidades de juego). La valoración se llevó a cabo en una escala tipo Likert de 5 puntos, siendo el 1 Nada de acuerdo y el 5 Totalmente de acuerdo. La escala utilizada obtuvo un valor en el coeficiente alfa de Cronbach de 0,876, el cual puede establecerse como aceptable.

En la parte de Actividad Física y Deporte se empleó el cuestionario PAQ-A de Martinez-Gomez et al. (2009). Este cuestionario se encuentra formado por 14 ítems que miden diferentes aspectos de la actividad física realizada por cada individuo mediante una escala de Likert de 5 puntos, utilizándose solo 13 ítems para calcular la Actividad Física Global y por niveles (Bajo, Medio y Alto) dependiendo del rango de Actividad que muestren los sujetos. En este cuestionario, hemos modificado el ítem 1 del cuestionario PAQ-A original para adaptarlo al alumnado correspondiente al cual hemos realizado el cuestionario para esta investigación. Se ha modificado de tal forma que hemos agrupado los deportes correspondientes del cuestionario original en "deportes individuales sin contacto (atletismo, tenis, ciclismo, etc)", "deportes individuales con contacto (karate, taekwondo, judo, etc)", "deportes colectivos (en equipo) sin contacto (voleibol, criquet, béisbol, 
etc)", "deportes colectivos con contacto (baloncesto, rugby, fútbol, etc)" y "no practico ningún deporte".

Por último, se empleó el cuestionario AD HOC. Se realizó este cuestionario para el registro de las variables de tipo sociodemográfico y las que estaban relacionadas con aspectos sobre género y edad.

\subsection{Procedimiento}

Se solicitó permiso al equipo directivo de los centros para poder asistir varios días a un colegio de Educación Primaria de la periferia de Granada, en este caso el "C.E.I.P. Manuel de Falla", situado en la población granadina de "Peligros", con el fin de aplicar los cuestionarios descritos a varios cursos de sexto (tercer ciclo). Todo esto se realizó para obtener los resultados sobre la relación entre la IE y la AF durante la etapa de Educación Primaria que permite desarrollar el objetivo de este Trabajo Fin de Grado.

Está investigación se llevó a cabo durante cuatro días de la semana en horario lectivo, a causa de que se solicitó la ayuda de los maestros de Educación Física para aplicar el cuestionario durante sus sesiones en respectivos cursos. El cuestionario se aplicó durante los primeros 20 minutos de cada clase de Educación Física en cada curso, y en todo momento se respetó el derecho de confidencialidad de todos los participantes, ya que el cuestionario fue totalmente anónimo.

\subsection{Análisis de los datos}

Para el análisis de los datos se empleó en software SPSS (v. 25) de IBM. Para ello se utilizaron frecuencias y medias para los descriptivos básicos, mientras que se emplearon comparación de medias (T de Student y ANOVA de un factor) para determinar la existencia de diferencias significativas en diferentes variables. El índice de confiabilidad fue fijado en un $95 \%$. También se emplearon correlaciones bivariadas de Pearson con el fin de determinar las relaciones entre variables. Para comprobar normalidad de los datos se comprobó la asimetría y curtosis de todos los ítems del cuestionario. Dado que no se obtuvo un valor superior a 2 en ambos, se concreta una distribución normal de la muestra en los ítems de la escala empleada. Dado que no se obtuvo un valor superior a 2 en ambos, se concreta una distribución normal de la muestra en los ítems de la escala empleada

\section{Resultados}

La tabla 1 representa los descriptivos de la realización de AF según el sexo, en la que observamos que los hombres realizan más AF semanalmente con una puntuación media de $\overline{\mathrm{X}}=$ 42,86 , en comparación con las mujeres que realizan menos con una puntuación media de $\bar{X}=36,73$. En conjunto, hombres y mujeres obtienen un valor de $t=3,103$ y una significatividad del 0,003, es decir, la AF según sexo presenta diferencias estadísticamente significativas al ser $P<0,05$.

Tabla 1. Actividad Física según sexo.

\begin{tabular}{clllll}
\hline Sexo & Media & Desviación estándar & $\mathbf{t}$ & Sig. \\
\hline \multirow{2}{*}{ AF } & Hombre & 42,86 & 7,42 & & \\
\cline { 2 - 6 } & Mujer & 36,73 & 10,12 & 0,003 \\
\hline
\end{tabular}

Nota: AF (Actividad Física Global)

La tabla 2 representa la media de descriptivos básicos de la IE según sexo del alumnado, donde observamos las medias de la IE Global dando a los hombres puntuaciones de $\bar{X}=$ 4,09 y a las mujeres puntuaciones de $\bar{X}=4,04$, con un valor conjunto entre hombre y mujeres de $\mathrm{F}=4,054$ y una significatividad del 0,047, es decir, que existirían diferencias estadísticamente significativas a ser $P<0,05$. El resto de medias con respecto a diferentes habilidades por las cuales nos vamos a guiar en la discusión de resultados significativos como son: PE (Percepción Emocional), GAE (Gestión Auto-Emocional) y GHE (Gestión Hetero-Emocional), no presentan diferencias estadísticamente significativas puesto que $P>0,05$. Sin embargo, UE (Utilización Emocional) presenta diferencias estadísticamente significativas.

Tabla 2. IE según sexo.

\begin{tabular}{|c|c|c|c|c|c|}
\hline & Sexo & Media & Desviación estándar & $\mathrm{F}$ & Sig. \\
\hline \multirow{2}{*}{ IEG } & Hombre & 4,09 & 0,39 & \multirow{2}{*}{4,054} & \multirow{2}{*}{0,047} \\
\hline & Mujer & 4,04 & 0,57 & & \\
\hline \multirow{2}{*}{ PE } & Hombre & 4,12 & 0,45 & \multirow{2}{*}{1,535} & \multirow{2}{*}{0,219} \\
\hline & Mujer & 4,25 & 0,60 & & \\
\hline \multirow{2}{*}{ GAE } & Hombre & 4,08 & 0,54 & \multirow{2}{*}{3,773} & \multirow{2}{*}{0,055} \\
\hline & Mujer & 3,93 & 0,72 & & \\
\hline \multirow{2}{*}{ GHE } & Hombre & 4,12 & 0,52 & \multirow{2}{*}{0,840} & \multirow{2}{*}{0,362} \\
\hline & Mujer & 4,04 & 0,62 & & \\
\hline \multirow{2}{*}{ UE } & Hombre & 3,92 & 0,63 & \multirow{2}{*}{4,587} & \multirow{2}{*}{0,035} \\
\hline & Mujer & 3,84 & 0,80 & & \\
\hline
\end{tabular}

Nota: IEG (Inteligencia Emocional Global); PE (Percepción Emocional); GAE (Gestión Auto-Emocional); GHE (Gestión Hetero-Emocional); UE (Utilización Emocional). 
La tabla 3 muestran los valores medios de la IE en función de las categorías de la AF. Esta se divide en 5 dimensiones de IE como son: IEG (IE Global); PE (Percepción Emocional); GAE (Gestión Auto-Emocional); GHE (Gestión HeteroEmocional); UE (Utilización Emocional), y en 3 categorías o niveles de AF como son "Baja", "Media" y "Alta". Como hemos estado viendo anteriormente nos centramos en los resultados obtenidos en la IE Global, observando así resultados como que la media de IEG es más alta cuando se realiza AF a un nivel alto con una puntuación media de $\bar{X}=4,34$, en comparación con la realización de AF a nivel medio, la cual obtiene una puntuación de $\overline{\mathrm{X}}=4,02$. Asimismo, cuándo se realiza AF a nivel bajo se obtiene la puntuación más baja con una media de $\bar{X}=3,92$. En su conjunto se obtiene un valor de $F=3,190$ y una significatividad del 0,046, por tanto, presenta diferencias estadísticamente significativas. Al contrario que IEG, el resto de dimensiones no presentan diferencias estadísticamente significativas al ser $P>0,05$.

Tabla 3. Actividad Física categorizada según Inteligencia Emocional

\begin{tabular}{|c|c|c|c|c|c|}
\hline & & Media & Desviación estándar & $\mathrm{F}$ & Sig. \\
\hline \multirow{4}{*}{ IEG } & Baja & 3,92 & 0,57 & \multirow{4}{*}{3,190} & \multirow{4}{*}{0,046} \\
\hline & Media & 4,02 & 0,49 & & \\
\hline & Alta & 4,34 & 0,35 & & \\
\hline & Total & 4,06 & 0,50 & & \\
\hline \multirow{4}{*}{ PE } & Baja & 4,01 & 0,69 & \multirow{4}{*}{2,073} & \multirow{4}{*}{0,132} \\
\hline & Media & 4,18 & 0,51 & & \\
\hline & Alta & 4,41 & 0,46 & & \\
\hline & Total & 4,19 & 0,54 & & \\
\hline \multirow{4}{*}{ GAE } & Baja & 3,93 & 0,71 & \multirow{4}{*}{1,560} & \multirow{4}{*}{0,216} \\
\hline & Media & 3,94 & 0,62 & & \\
\hline & Alta & 4,26 & 0,64 & & \\
\hline & Total & 3,99 & 0,65 & & \\
\hline \multirow{4}{*}{ GHE } & Baja & 3,96 & 0,56 & \multirow{4}{*}{2,107} & \multirow{4}{*}{0,128} \\
\hline & Media & 4,03 & 0,60 & & \\
\hline & Alta & 4,34 & 0,40 & & \\
\hline & Total & 4,07 & 0,58 & & \\
\hline \multirow{4}{*}{ UE } & Baja & 3,68 & 0,69 & \multirow{4}{*}{2,662} & \multirow{4}{*}{0,076} \\
\hline & Media & 3,83 & 0,76 & & \\
\hline & Alta & 4,25 & 0,55 & & \\
\hline & Total & 3,87 & 0,73 & & \\
\hline
\end{tabular}

Nota: IEG (Inteligencia Emocional Global); PE (Percepción Emocional); GAE (Gestión Auto-Emocional); GHE (Gestión Hetero-Emocional); UE (Utilización Emocional)

Por último, la tabla 4 muestra las correlaciones entre el nivel de Actividad Física y la puntuación media entre cada dimensión de la IE y la IEG. Para comentar esta tabla nos vamos a centrar únicamente en la primera fila, donde vamos a observar una serie de resultados en las que aparecen unos asteriscos, dependiendo del resultado uno o dos asteriscos.
Esto significa que, ${ }^{*} \mathrm{p}<0,05$; $^{* *} \mathrm{p}<0,01$, es decir, de mayor fortaleza de correlación a menor. La correlación entre AF e IEG obtuvo la mayor fortaleza de correlación $(r=0,351$; $\mathrm{p}<0,01)$, seguido de UE $(\mathrm{r}=0,293 ; \mathrm{p}<0,01), \mathrm{PE}(\mathrm{r}=0,289$; $\mathrm{p}<0,01)$, GAE $(\mathrm{r}=0,274 ; \mathrm{p}<0,05)$, por último, GHE $(\mathrm{r}=$ $0,272 ; \mathrm{p}<0,05)$. 
Tabla 4. Nivel de Actividad Física según inteligencia.

\begin{tabular}{cccccc}
\hline & IEG & PE & GAE & GHE & UE \\
\hline AF & $0,351^{* *}$ & $0,289^{* *}$ & $0,274^{*}$ & $0,272^{*}$ & $0,293^{* *}$ \\
IEG & & $0,831^{* *}$ & $0,770^{* *}$ & $0,897^{* *}$ & $0,684^{* *}$ \\
PE & & $0,526^{* *}$ & $0,676^{* *}$ & $0,481^{* *}$ \\
GAE & & & $0,558^{* *}$ & $0,358^{* *}$ \\
GHE & & & & $0,548^{* *}$ \\
\hline
\end{tabular}

Nota 1: AF (Actividad Física Global); IEG (Inteligencia Emocional Global); PE (Percepción Emocional); GAE (Gestión Auto-Emocional); GHE (Gestión Hetero-Emocional); UE (Utilización Emocional).

Nota $2: *, \mathrm{p}<0,05 ;{ }^{* *}, \mathrm{p}<0,01$.

\section{Discusión}

Este estudio se llevó a cabo con 86 estudiantes de Educación Primaria de un colegio de la periferia de Granada, en concreto con C.E.I.P. Manuel de Falla de la población de Peligros. El objetivo principal del presente estudio consistió en analizar las relaciones entre la práctica de actividad física, la inteligencia emocional y diferentes factores sociodemográficos. De este modo, algunos estudios de carácter similar son los realizados por De Benito (2013), García-Coll, GrauperaSanz, Ruiz-Pérez y Palomo-Nieto (2013), Merchán, Bermejo y González (2014) o Ruiz, Lorenzo y García (2013).

En cuanto a la realización de actividad física según el sexo, observamos diferencias estadísticamente significativas, en el cual, los hombres poseen puntuaciones superiores en la realización de actividad física, aun considerando que el porcentaje global de mujeres estudiadas fue superior al de los hombres. Esto se puede deber a que generalmente los hombres suelen realizar más actividad física que las mujeres, o incluso que en estas edades los hombres son más activos que las mujeres puesto que se involucran más en la práctica deportiva. Según Álvarez-Bogantes (2016), cabe recalcar las preferencias de las actividades de nińos y niñas en el deporte escolar, donde los niños prefieren involucrarse en actividades de moderada intensidad a vigorosa.

En los resultados de los descriptivos obtenidos en las dimensiones de la IE según el sexo se observa como únicamente encontramos diferencias estadísticamente significativas entre hombres y mujeres en la dimensión UE, ya que los hombres en esta dimensión tienen una media superior a las de las mujeres. Esto se puede deber a que los chicos utilizan las emociones mejor que las chicas, por estar más en contacto con la práctica deportiva tanto dentro como fuera de la escuela, y por tanto en constante relación con diferentes compañeros y otros. Los alumnos que practican deportes colectivos tienen mayores niveles de Inteligencia Emocional que los alumnos que sólo realizan Educación Física en el instituto (De Benito, 2013). Siguiendo argumentos de Álvarez-Bogantes (2016), los niños tienen mayor implicación con la práctica del fútbol y deportes colectivos en general, por lo que los incita a involucrarse más en la actividad física. Por lo que trabajar y ejercitar con el alumnado la práctica de actividad física genera una enseńanza adecuada que facilite el desarrollo de diferentes emociones y su conocimiento (Ruíz, Lorenzo y García, 2013). Con respecto a las demás dimensiones no se muestran diferencias estadísticamente significativas entre hombre y mujeres.

En los resultados obtenidos entre las cinco dimensiones de la IE y los diferentes niveles de actividad física, observamos la existencia diferencias estadísticamente significativas con respecto a IEG. Esto es así, ya que, observamos que las media obtenidas entre los diferentes niveles de actividad física son mayores entre ellas en comparación con las demás dimensiones de la IE. Este valor estadísticamente significativo de la IEG, se puede deber a que cuando se realiza actividad física a nivel medio y alto se fortalece y se interacciona más emocionalmente, como identificando y reconociendo emociones (PE), gestionando y controlando emociones propias (GAE) y diferentes (GHE) y saber utilizar diferentes emociones (UE). Este valor significativo obtenido englobaría las cuatro dimensiones anteriores, por lo que, como hemos comentado anteriormente al realizar actividad física media o alta se favorece e incrementan estas cuatro dimensiones. En un estudio similar a este se concluye que la IE obtiene un coeficiente de consistencia interna elevado, por lo que la IE resulta adecuada para su aplicación general en deportistas e, incluso, para tomar decisiones diagnósticas en psicología del deporte (García-Coll, Graupera-Sanz, Ruiz-Pérez y Palomo-Nieto, 2013).

En la última tabla entre las correlaciones entre niveles de actividad física y la media de cada dimensión de IE, se observa que AF e IEG muestran correlación con mayor fortaleza que las demás. Esto se puede originar así, por el simple motivo que la $\mathrm{AF}$ está relacionada con las cuatro dimensiones de IE, por lo que cuanta más AF se realice más favorecerá las IEG y sus cuatro dimensiones. También observamos como la correlación de menor fortaleza es AF y GHE, y esto se puede deber a que el alumnado cuándo se encuentra realizando algún tipo de $\mathrm{AF}$, no maneja ni controla las emocio- 
nes de sus compañeros, sino que seguramente se encuentren centrados en la práctica deportiva en ese momento. Según Calero (2012), la asignatura de Educación Física tiene una implicación muy directa con los elementos que componen la inteligencia emocional, ya que la empatía, el control de las emociones, la automotivación y las habilidades sociales son elementos que necesariamente van a existir dentro de la práctica deportiva, ya sea a nivel competitivo o a nivel educativo. Por otro lado, De Benito (2013), afirma una mejor Percepción Emocional (PE), en adolescentes de 11-20 años, que practican Actividad Física y Deporte más continuamente, pero sobre todo afirma haber una mayor PE en los momentos de práctica deportiva, y así favorecer la percepción relacional, de apoyo, mayor motivación, mayor autonomía, etc.

En este estudio realizado entre las relaciones entre la IE y AF podemos encontrar limitaciones, no cuantiosas pero mejorables. La limitación más importante del estudio realizado es sobre todo la falta de tiempo, puesto que este estudio se puede realizar con más tranquilidad y destreza si se tuviera tiempo suficiente. Al habla de la limitación de falta de tiempo también encontramos limitaciones como que el estudio podría haber abarcado más, ya que, la muestra es bastante pequeña $(n=86)$, si este estudio se hubiera realizado con más sujetos, hubiéramos obtenido resultados más fiables y significativos. La limitación de la compatibilidad horaria también ha sido un factor importante, puesto que ha habido momentos que no se podían encontrar para realizar diferentes acciones del estudio. Por último, tenemos la limitación de la falta de comprensión por parte del alumnado y con lo que respecta al cuestionario, porqué, aunque se aclarasen todo tipo de dudas y se explicara cada parte del cuestionario durante el proceso de aplicación, siempre existen ciertos aspectos que se escapan a la comprensión.

\section{Conclusiones}

La etapa de Educación Primaria es una etapa primordial para fomentar la IE, ya que, con una actuación activa y adecuada va a ser posible un incremento de la motivación, autonomía, relaciones sociales, y por lo general, de un bienestar globalizado por parte de cada alumno/a (De Benito, 2013). En este estudio llevado a cabo en escolares de tercer ciclo de Educación Primaria, concluimos que este alumnado percibe mejor las emociones (PE), que cualquiera de las otras dimensiones durante la práctica deportiva. También vemos como la realización de AF ayuda a aclarar y fortalecer las diferentes dimensiones de la IE, sobre todo en el alumnado que realiza AF a nivel medio o alto donde hemos observado los resultados más significativos. Por lo tanto, podemos afirmar que la IE se relaciona bastante con la $\mathrm{AF}$, sobre todo tienen mucho en común en lo académico, pudiéndose trabajar la IE perfectamente en el área de EF, y de forma significativa.

Por otro lado, podemos decir que los hombres normalmente suelen ser más activos e incitados por la AF, sin embargo, las mujeres saben reconocer y gestionar mejor las emociones que los hombres aún sin ser tan activas como ellos en la AF. Aunque los hombres saben utilizar mejor las emociones que las mujeres, y esto es debido seguramente a la conveniencia de los hombres sobre la AF y a su mayor actividad en la práctica deportiva. Por último, hemos observado como el compañerismo del grupo-clase y el buen clima es esencial para reconocer las diferentes dimensiones de la IE durante la práctica deportiva en EF, y sobre todo muy contradictorio los problemas de conducta que puedan surgir en el grupo-clase.

\section{Referencias}

1. Alzina, R. B. (2005). La educación emocional en la formación del profesorado. Revista Interuniversitaria de Formación del Profesorado, 54, $95-$ 114.

2. Arango-Caramelo, M. P. (2017). Inteligencia emocional en niños de Educación Primaria: programa de intervención (Trabajo Fin de Grado).

3. Bogantes, C. Á. (2016). Entendiendo los factores que determinan la actividad física en el entorno escolar desde la perspectiva de los nińos y niñas. MHSALUD: Revista en Ciencias del Movimiento Humano y Salud, 13(1), 1-17.

4. Conde, C., y Almagro, B. J. (2015). Estrategias para desarrollar la inteligencia emocional y la motivación en el alumnado de educación física. E-motion: Revista de Educación, Motricidad e Investigación, 1, 212-220.

5. De Benito, M. M. (2013). Análisis de un modelo estructural de inteligencia emocional y motivación autodeterminada en el deporte. (Tesis doctoral). Universitat de València.

6. Di Fabio, A., y Kenny, M. E. (2016). Promoting well-being the contribution of emotional intelligence. Frontiers in Psychology, 7, 1182.

7. Duran, C., Lavega, P., Salas, C., Tamarit, M., e Invernó, J. (2015). Educación Física emocional en adolescentes. Identificación de variables predictivas de la vivencia emocional. Cultura, Ciencia y Deporte, 10(28), 5-18.

8. García-Coll, V., Graupera-Sanz, J. L., Ruiz-Pérez, L. M., y PalomoNieto, M. (2013). Inteligencia emocional en el deporte: Validación española del Schutte Self Report Inventory (SSRI) en deportistas españoles. Cuadernos de Psicología del Deporte, 13(1), 25-36.

9. Lleixà, T. (2017). Didáctica de la Educación Física: Nuevos temas, nuevos contextos. Didáctica: Revista de Investigación en Didácticas Especificas, 2, 2-5.

10. Mateos, M. E. (2012). La inteligencia emocional en el área de Educación Física. La peonza: Revista de Educación Física para la Paz, (7), 65.

11. Martínez-Gómez, D., Martínez-de-Haro, V., Pozo, T., Welk, G. J., Villagra, A., Calle, M. E., ... y Veiga, O. L. (2009). Fiabilidad y validez del cuestionario de actividad física PAQ-A en adolescentes españoles. Revista Española de Salud Pública, 83, 427-439.

12. Merchán, I. M., Bermejo, M. L., y de Dios González, J. (2014). Eficacia de un Programa de Educación Emocional en Educación Primaria. Revista de Estudios e Investigación en Psicología y Educación, 1(1), 91-99.

13. OMS (2019). Salud mental. Recuperado de https://www.who.int/es/ 
news-room/facts-in-pictures/detail/mental-health

14. Ozcorta, E. J. F., Torres, B. J. A., y Buńuel, P. S. L. (2015). Inteligencia emocional percibida y el bienestar psicológico de estudiantes universitarios en función del nivel de actividad física. Cultura Ciencia Deporte, 10(28), 31-39.

15. Puertas-Molero, P., González-Valero, G., y Sánchez-Zafra, M. (2017) Influencia de la práctica físico deportiva sobre la Inteligencia Emocional de los estudiantes: Una revisión sistemática. ESHPA, 1(1), 10-24.

16. Rosa, M. (1992). Historia de la educación física. Cuadernos de Sección. Educación, 5, 27-47.

17. Ruiz, G., Lorenzo, L., y García, Á. (2013). El trabajo con la inteligencia emocional en las clases de educación física: valoración de una experiencia piloto en educación primaria. Journal of Sport and Health Research, 5(2), 1-4.

18. Salovey, P., y Mayer, J. D. (1990). Emotional intelligence. Imagination, Cognition and Personality, 9(3), 185-211.

19. Zurita-Ortega, F., Moreno-Arrebola, R., González-Valero, G., VicianaGarófano, V., Martínez-Martínez, A. y Muros, J. J. (2018). Revisión conceptual de la conexión entre inteligencia emocional y autoconcepto físico. SPORT TK-Revista EuroAmericana de Ciencias del Deporte, 7(1), 139-144. 